\title{
ACTION OF BLEOMYCIN ON PROLIFERATING PLANT CELLS
}

\author{
M. E. Fernández-Gómez and C. DE LA Torre \\ Departamento de Citología, Instituto de Biología Celular (C.S.I.C.), \\ Velázquez, 144. Madrid-6, Spain
}

(Received for publication June 7, 1976)

\begin{abstract}
Bleomycin $\left(10^{-6} \mathrm{M}\right)$ has been tested in Allium cepa L. meristems which are formed by a proliferating cell population growing under steady state conditions. Chromosome breaks were apparently induced by the antibiotic in cells in $\mathrm{G}_{2}$ period since anaphases with chromatid breaks were formed at a time shorter than $\mathrm{G}_{2}+$ prophase duration. Stimulation of entrance of $\mathrm{G}_{2}$ cells into mitosis is suggested both by an increase in the frequency of early prophases and by the study of waves of prophases in a synchronous subpopulation labelled by caffeine. Progression of other mitotic phases was unaffected. Nucleologenesis rate was increased by the antibiotic in a fashion resembling protein synthesis inhibitors. Protein synthesis is inhibited by $10^{-6} \mathrm{M}$ bleomycin to the same extent as $4 \times 10^{-8} \mathrm{M}$ anisomycin. Both facts suggest that bleomycin has a direct inhibitory effect on protein synthesis in meristems.

Given the nucleologenesis sensitivity to nucleolar RNA inhibition it is suggested that the antibiotic activity on nucleolar transcription is mediated through DNA.
\end{abstract}

Root meristems are ideal tissues to study the effect of antitumor drugs on cycling cells, since under fixed environmental conditions, linear kinetics of growth is attained, and the basic molecular changes are similar to those taking place in any other eukaryotic proliferating cell ${ }^{12}$.

The linear kinetics in plant meristems is sustained by the fact that the number of cycling cells in the tissues remains constant with time, since half of the newly formed cells abandon the meristem towards the differentiation zone of the root every cycle time. Besides, with these kinetics the number of meristem cells in any cycle compartment remains constant with time and is directly proportional to its duration (in time units) ${ }^{10)}$. Hence, the action of an external agent can be evaluated either by recording the change in frequency of cells in a cycle compartment or by direct measurement of the timing of a process, if a label for a specific cycle compartment is used.

The action of bleomycin on interphase as well as chromosomal and nucleolar phases around mitosis is being analyzed. An apparent stimulation in entering prophase as well as an increase in rate of nucleologenesis are found in the bleomycin-treated meristems.

\section{Materials and Methods}

The material used was root meristems of Allium cepa L. bulbs growing in filtered tap water, under continuous air bubbling, at $15^{\circ} \mathrm{C}$ and darkness.

Treatments

The roots still attached to the bulbs were treated either with $10^{-6} \mathrm{M}$ bleomycin or $4 \times 10^{-6} \mathrm{M}$ anisomycin in tap water.

Cytological procedures

For the nuclear studies twelve roots of 3 repeated experiments were fixed in a $3: 1$ ethanol-acetic acid mixture. They were stained by acetic orcein and squashes of the terminal $2 \mathrm{~mm}$ of the roots were made according to the TJIO and LEVAN technique ${ }^{17}$.

For nuclear studies, roots were fixed in formalin-hydroquinone, silver impregnated by a $\mathrm{AgNO}_{3}$ solution and finally were squashed ${ }^{1}$. 
Labelling of a synchronous cell subpopulation within the meristem

Caffeine selectively inhibits the formation of the cell plate in those cells that are progressing through their telophase during treatment. Caffeine therefore "labels" a synchronous population of cells by rendering them binucleate ${ }^{4}$.

The roots still attached to the bulbs were treated with $0.1 \%$ caffeine in tap water for 1 hour.

Protein synthesis measurement

Bulbs were incubated with $0.02 \mu \mathrm{Ci} / \mathrm{ml} 10^{-4} \mathrm{M}^{14} \mathrm{C}$-leucine (spec. act. $344 \mu \mathrm{Ci} / \mathrm{mM}$ ). They were considered as control bulbs, while others were incubated in the same way in anisomycin.

Fifty to sixty root tips $(4 \mathrm{~mm})$ were removed at the end of 3 hours and homogenized with alumina. At the end of the different periods, 50 60 more roots were removed and the same procedure followed. Each extract was diluted with Tris-(hydroxymethyl) amino-methane buffer at $\mathrm{pH} 7.5$ and finally centrifuged for 15 minutes at $15,000 \mathrm{~g}$ in order to pellet the alumina. et al ${ }^{11}$.

The protein content $(\mathrm{mg} / \mathrm{ml})$ was determined in an aliquot of the supernatant according to LowRY

Another aliquot was treated for 10 minutes with $20 \%$ trichloroacetic acid plus $0.1 \mathrm{~m}$ leucine at room temperature. Afterwards it was heated for 15 minutes at $85 \sim 95^{\circ} \mathrm{C}$. The extract was then filtered (through Whatman GF/50 Filter), washed with $5 \%$ trichloroacetic acid plus $40 \mathrm{~mm}$ leucine and assayed in a glas-flow counter for incorporated radioactivity $(\mathrm{cpm})$. The concentration $(\mathrm{mg})$ of the ${ }^{14} \mathrm{C}$-leucinelabelled protein $(\mathrm{cpm})$ was also determined.

\section{Results}

The percentage of cells at the different mitotic phases (pro-, meta-, ana- and telophase) were recorded as well as the percentage of cells in the different phases of the nucleolar distribution cycles, in control meristems and in meristems treated for 6 hours with bleomycin. These counts have been used to represent the proportional timing of the corresponding phases in Fig. 1. After treatment the mitotic index remained fairly unchanged and about $1 / 3$ anaphases show chromosomes with breaks in their arms. This effect on chromosomes has been extensively described ${ }^{7,8)}$ and was not further investigated in our work.

\section{Matching of Chromosomal and Nucleolar Cycles}

Matching can be accomplished by the fact that the prophase/metaphase boundary exactly coincides with the disorganization of nucleoli, and in untreated cells starts by mid-prophase (Fig. 1). The nucleolus has disappeared in metaphase. Reorganization of new nucleolus takes place during a cycle segment corresponding to telophase and early $\mathrm{G}_{1}$.

Six hours after bleomycin treatment was initiated, the matching of both chromosomal and nucleolar

Fig. 1. Matching of chromosomal and nucleolar cycle in mitosis has been accomplished by taking into account the observation that end of nucleolar disorganization always took place at the end of prophase.

Control

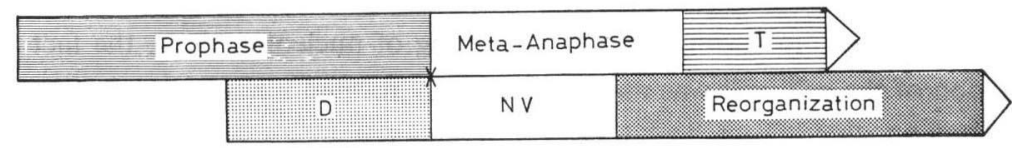

Bleomycin

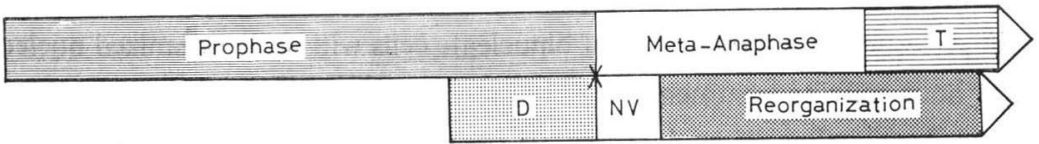


cycles evidences an increase in the percentage of early prophases, while the number of cells in the phase of nucleolar disorganization has diminished. Unexpectedly nucleologenesis is advanced in relation to telophase in bleomycin-treated meristems.

\section{Mitosis Entrance}

To analyze whether the larger number of cells in early prophases is due to lengthening of this period or to a greater entrance of premitotic $\left(G_{2}\right)$ cells into mitosis, binucleate cells were used. The binucleate cells show prophase of their next mitosis as shown in Fig. 2.

The earliest biprophase in the control meristems are recorded at the 22 nd hour after caffeine, confirming earlier data ${ }^{\text {})}$. Continuous bleomycin treatments were initiated at times corresponding to either the $23 \mathrm{rd}, 24$ th or 25 th hour after caffeine and the numbers of prophases were recorded at hourly intervals. A larger number of prophases is recorded than in control meristems when bleomycin is provided at the $23 \mathrm{rd}$ and 24th hour but not at the 25th hour or later (which are not shown since they were similar to control values). Metaphase waves always resembled their corresponding prophase waves, so the bleomycin-induced increase in prophases seems to be due to an increase in mitosis entrance, i.e. shortening of some premitotic requirement whose location is not farther advanced than 3 hours before mitosis. In our experimental conditions $G_{2}$ period lasts 4.8 hours while prophase takes 1.8 hours ${ }^{\theta, \theta)}$.

\section{Nucleologenesis Timing in Synchronous Cells}

With the aim of measuring directly the timing of nucleologenesis, the fact that caffeine prevents the formation of the cell wall was used. The newly formed binucleate cells are naturally synchronous cells which are labelled as such during their telophase and, hence, initiating $G_{1}$ of a new cycle inmediately after the labelling period (Fig. 3). As nucleologenesis occurs at these stages, the timing of nucleologenesis can be obtained by simply estimating the number of binucleate cells with fully organized nucleoli at different times after the 1 hour caffeine treatment.

As Fig. 4 shows, the mean time of nucleologenesis (time when $50 \%$ of the binucleate cells have
Fig. 2. Bleomycin action in prophase.

A synchronous cell population, labelled as binucleate by caffeine, has been studied at times .

Bleomycin starts at 23rd hour.

C: Control and bleomycin at 25 th hour.

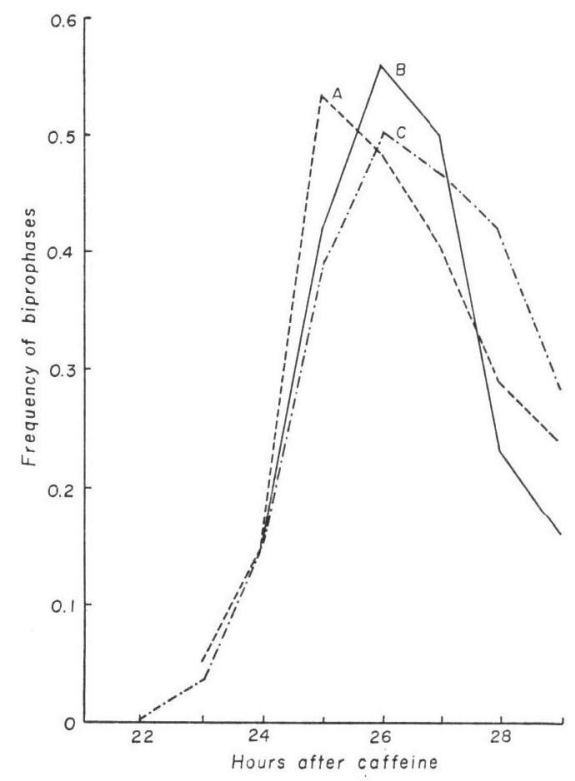

Fig. 3. Cytological method to measure rate of nucleologenesis in a direct way.

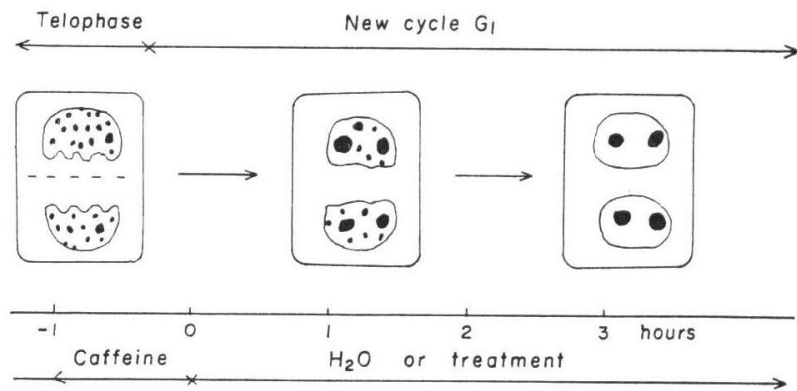


Fig. 4. Frequency of binucleate cells with fully organized nucleoli after the 1-hour caffeine treatment (in control cells) and after 1-hour caffeine + bleomycin and then bleomycin in continuous treatment.

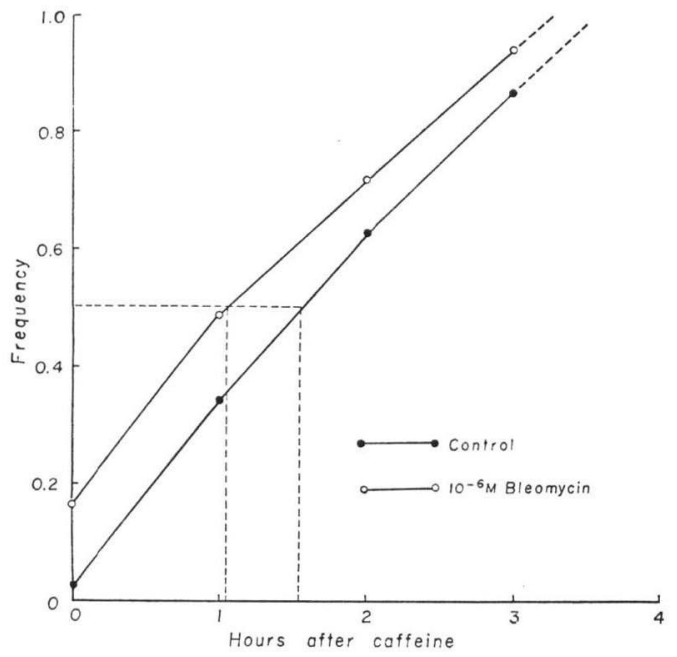

Fig. 5. Rates of protein synthesis in meristems growing under either $4 \times 10^{-6} \mathrm{M}$ anisomycin or $10^{-6} \mathrm{M}$ bleomycin.

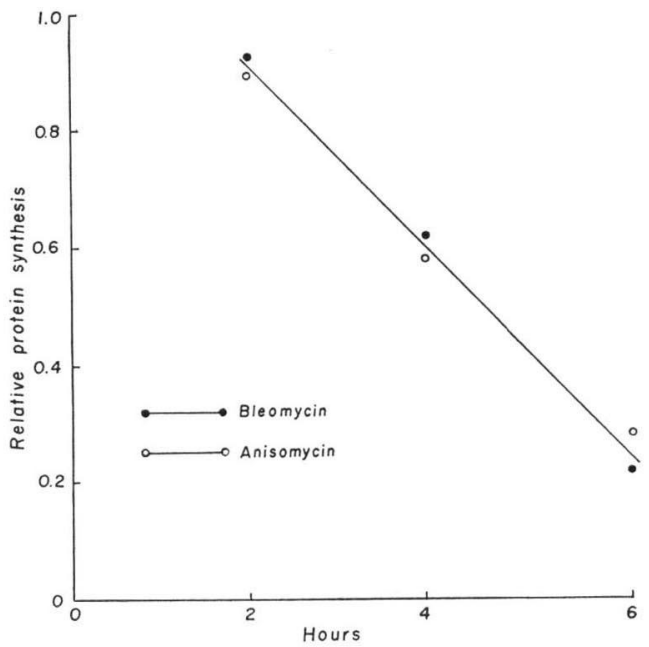

fully organized nucleoli) is shorter in the presence of bleomycin.

The speeding up of nucleologenesis greatly resembles the situation occurring in similar cells when simultaneous protein synthesis was inhibited ${ }^{5)}$.

\section{Rate of Protein Synthesis}

In order to study whether $10^{-8} \mathrm{M}$ bleomycin had any effect on protein synthesis, the rate was evaluated and compared to the rate of protein synthesis in control meristems growing under steady state conditions as well as with the rate in meristems growing under anisomycin treatment, a reported inhibitor of protein synthesis in eukaryotic cells.

Fig. 5 shows that bleomycin induces a reduction in the rate of protein synthesis which is similar to that produced by anisomycin.

\section{Discussion}

The maintenance of mitotic index at the 6th hour after initiating treatment shows that $10^{-6} \mathrm{M}$ bleomycin does not prevent cells from entering mitosis. It seems even that there is an increased entrance of $\mathrm{G}_{2}$ cells into prophase, since many more early prophases are recorded than in control meristems. Bleomycin is seen to induce prophase entrance of mononucleate and binucleate cells located in $2 \sim 3$ hours previous to mitosis. These advanced cells seem to progress normally throughout their prophase.

However, NAGATSU et al., ${ }^{13,14)}$; HITTELMAN and RAO ${ }^{7)}$ and WATANABE et al., ${ }^{10)}$ report a bleomycininduced arrest in $\mathrm{G}_{2}$ in mammalian cells, but the concentration of bleomycin they used is much higher than the concentration here tested. NAGATSU et al. ${ }^{14)}$ report a leap from $\mathrm{G}_{2}$ to the next $\mathrm{S}$ period, i.e. the bleomycin inductor of endoreduplication, which we do not have any evidence for.

Since chromosomal breaks are observed in $1 / 3$ of anaphases as early as 6 hours (actually $25 \%$ of anaphases at the 4 th hour after bleomycin also had chromosome breaks) and since $\mathrm{G}_{2}+$ prophase timing is 6.6 hours in experimental conditions, we can infer that bleomycin must directly induce chromatid breaks in $\mathrm{G}_{2}$ chromosomes and they are made visible in their first mitosis. A portion of the $\mathrm{G}_{2}$ cells 
stimulated to enter prophase could well have strand breaks. This response resembles bleomycin action reported in cultured mammalian cells chromosomes by PAIKA et al., ${ }^{15)}$. We have not further investigated the kinetics of appearance of micronuclei through lagging broken chromosome arms, since the bleomycin capacity to damage DNA in its replicating period is extensively documented ${ }^{8,16)}$.

The progression of some of the mitotic phases and of the phases of the nucleolar cycle in root meristems depends on simultaneous macromolecule biosynthesis. In our experiments bleomycin is seen to induce shortening of nucleologenesis in relation of telophase, when compared with untreated meristem (Fig. 1).

Moreover, the use of caffeine labelling allows one to measure the shortening of the nucleologenesis timing induced by bleomycin, which resembles the shortening of nucleologenesis produced by simultaneous inhibition of protein synthesis ${ }^{5)}$. Such differential sensitivity to bleomycin in plant nucleologenesis resembled the response pattern to protein synthesis inhibition ${ }^{2,5}$.

The acceleration of nucleologenesis if brought about through the simultaneous protein synthesis inhibition could lead us to postulate a direct action of this antibiotic on protein synthesis. It would be difficult to relate it to the primary damage of DNA as WATANABE et al., ${ }^{18,18)}$ leave open when studying the response of DNA, RNA and protein syntheses to bleomycin. However, bleomycin does not seem to have any effect on either prophase or metaphase progression even though it inhibits the normal rate of protein synthesis in control meristems by $70 \%$, and it has been shown that progression of mitotic condensation in prophase chromosomes in Allium depends on protein synthesis taking place in prophase itself ${ }^{3)}$.

Finally, the nucleologenesis test in Allium demonstrates that bleomycin has no direct action on nucleolar transcription, since this process is fully dependent on simultaneous rRNA and was not impeded at all by the antibiotic.

\section{References}

1) Fernández-Gómez, M. E.; J. C. Stockert, J. F. Lopez-Saez \& G. Gimenez-Martin： Staining plant cell nucleoli with $\mathrm{AgNO}_{3}$ after formalin-hydroquinone fixation. Stain Techn. 44: 48 49, 1969

2) FernándeZ-Gómez, M. E.; C. DE LA TORRe \& G. Gimenez-Martin: Accelerated nucleolar reorganization with shortened anaphase and telophase during cycloheximide inhibition of protein synthesis in onion root cells. Cytobiologie 5: 117 124, 1972

3) Garcia-Herdugo, G.; M. E. Fernández-Gómez, J. Hidalgo \& J. F. Lopez-Saez: Effects of protein synthesis inhibition during plant mitosis. Exptl. Cell Res. 89: 336 342, 1974

4) Gimenez-Martin, G.; A. Gonzalez-Fernandez \& J. F. Lopez-Saez: A new method of labelling cells. J. Cell Biol. 26: 305 309, 1965

5) Gimenez-Martin, G.; C. De la Torre \& M. E. Fernandez-Gomez: Experimental analysis of nucleolar reorganization. J. Cell Biol. 60: 502 507, 1974

6) Gonzalez-Fernandez, A.; G. Gimenez-Martin \& C. DE LA Torre: The duration of the interphase periods at different temperatures in root tip cells. Cytobiologie 3:367 371, 1971

7) Hittelman, W. N. \& P. N. Rao: Bleomycin-induced damage in prematuraly condensed chromosomes and its relationship to cell cycle progression in CHO cells. Cancer Res. 34:3433 3439, 1974

8) Kürten, S. \& G. OвE: Premature chromosome condensation in the bone marrow of Chinese hamster after application of bleomycin in vivo. Mutation Res. 27: 285 294, 1975

9) Lopez-Saez, J. F.; G. Gimenez-Martin \& A. Gonzalez-Fernandez: Duration of the cell division cycle and its dependence on temperature. Z. Zellforch. 75: 591 600, 1966

10) Lopez-Saez, J. F.; A. Gonzalez-Fernandez, C. de la Torre, J. L. Diez, M. E. Fernandez-Gomez, M. Hernandez-Navarrete, G. Garcia-Herdugo \& G. Gimenez-Martin: A model for cell cycle and growth kinetics in roots. J. Theor. Biol. 53: 463 473, 1975

11) Lowry, O. H.; N. J. Rosebrough, A. L. Farr \& R. J. Randall: Protein measurements with the Folin phenol reagent. J. Biol. Biochem. 193: 265 275, 1951

12) Mrtchison, J. M.: The Biology of the Cell Cycle. Cambridge Univ. Press, 1971

13) Nagatsu, M.; T. Okagaki, R. M. Richart \& A. Lambert: Effects of bleomycin on nuclear DNA in transplantable VX-2 carcinoma of rabbit. Cancer Res. 31: 992 996, 1971

14) Nagatsu, M.; R. M. Richart \& A. Lambert: Effects of bleomycin on the cell cycle of EhrLich ascites carcinoma. Cancer Res. 32: 1966 1970, 1972 
15) PAIKA, K. D. \& A. KRISHAN: Bleomycin-induced chromosomal aberrations in cultured mammalian cells. Cancer Res. 33: 961 965, 1973

16) Takita, T.; J. Muraoka, T. Yoshioka, A. Fuji, K. Maeda \& H. Umezawa: The chemistry of bleomycin. IX. The structures of bleomycin and phleomycin. J. Antibiotics 25: 755 758, 1972

17) Tuo, J. H. \& A. Levan: The use of oxiquinoline in chromosome analysis. Ann. Estc. Exptl. Aula Dei 2: $21 \sim 64,1950$

18) Watanabe, M.; Y. Takabe, T. Katsumata, T. Terashima \& H. Umezawa: Response in macromolecular synthesis of mouse L cells to bleomycin, with special reference to cell-antibiotic interaction. J. Antibiotics 26: $417 \sim 423,1973$

19) Watanabe, M.; Y. Takabe, T. Katsumata \& T. Terasima: Effects of bleomycin on progression through the cell cycle of mouse L-cells. Cancer Res. 34: 878 881, 1974 